\title{
Traçado Farmacoepidemiológico dos pacientes admitidos no Pronto-Socorro de um Hospital do interior de Minas Gerais
}

\author{
Pharmacoepidemiological Tracing of patients admitted to the Emergency Room of a Hospital in the \\ interior of Minas Gerais
}

Trazabilidad Farmacoepidemiológica de pacientes ingresados en Primeros Auxilios de un Hospital del interior de Minas Gerais

Recebido: 23/05/2021 | Revisado: 31/05/2021 | Aceito: 04/06/2021 | Publicado: 18/06/2021

\author{
Allan Kardek das Virgens Souza Junior \\ ORCID: https://orcid.org/0000-0001-7210-6523 \\ Faculdade Independente do Nordeste, Brasil \\ E-mail: kardekjunior@gmail.com \\ Matheus Santos Marques \\ ORCID: https://orcid.org/0000-0001-9734-1001 \\ Faculdade Independente do Nordeste, Brasil \\ E-mail: matheusmarques@fainor.com.br
}

\begin{abstract}
Resumo
O presente estudo tem o objetivo de mensurar e apresentar dados epidemiológicos que possam contribuir na gestão de um hospital do interior de Minas Gerais, com intuito de aprimorar a qualidade dos serviços oferecidos. Trata-se de um estudo com delineamento observacional do tipo transversal, retrospectivo com abordagem quali-quantitativa. $\mathrm{O}$ hospital é de pequeno porte, contendo 46 leitos. A técnica de amostragem utilizada é a não probabilística por conveniência. A ferramenta Excel versão 2007 foi utilizada para coleta e análise dos dados. As informações foram extraídas mediante as variáveis: Idade, sexo, motivo da admissão, medicamentos utilizados e desfechos. A amostra analisada contém 9.246 fichas de admissão para o pronto- atendimento. Dentre estas, foram incluídas 1.328 (14,36\%) e, excluídas $7.918(85,64 \%)$. O sexo feminino é maioria no espaço amostral, (61,39\%), desigualmente o sexo masculino representa $(38,60 \%)$. A idade de maior frequência está entre 0 a 10 anos de $(19,14 \%), 11$ a 20 anos $(12,88 \%)$ e, 41 a 50 anos $(15,06 \%)$. As queixas mais comuns são: Infecções das Vias Aéreas Superiores (IVAS), com uma média relativa total de 15,64\%; Lombalgia 10,35\%; Crise asmática 5,69\%. Os medicamentos mais utilizados são; Dipirona média relativa total de 17,33\%; Dexametasona 12,06\%; Diclofenaco 8,74\%. A média relativa total da taxa de liberação é de $95,80 \%$, por sua vez, a média relativa total de internações é de $4,20 \%$. Conclui-se que, conhecer o traçado farmacoepidemiológico de um ambiente hospitalar é poder fornecer a todo corpo de funcionários o perfil de pacientes acolhidos, tal qual também, serve de base para identificação e análise das reais necessidades por parte dos gestores. Entretanto é necessário salientar que o estudo em questão abre caminhos para a construção de futuros trabalhos, direcionado ao entendimento, quanto à frequência de retorno dos mesmos ao pronto-socorro, referindo as mesmas queixas, bem como o percentual de pacientes liberados, com terapia medicamentosa prescrita para posterior aquisição a nível ambulatorial.
\end{abstract}

Palavras-chave: Farmacoepidemiologia; Variáveis; Hospital; Gestores.

\begin{abstract}
This study aims to measure and present epidemiological data that can contribute to the management of a hospital in the interior of Minas Gerais, in order to improve the quality of services offered. This is a retrospective, cross-sectional observational study with a qualitative-quantitative approach. The hospital is small, containing 46 beds. The sampling technique used is non-probability for convenience only. The MS Excel tool, version 2007, was used for data collection and analysis. The information was extracted using the following variables: Age, gender, reason for admission, medications used, and outcomes. The sample analyzed contains 9.246 admission forms for the emergency room. Among the samples, 1.328 were included $(14.36 \%)$ and 7.918 were excluded $(85.64 \%)$. The female gender is the majority in the sample space, $(61.39 \%)$, while the male gender represents $(38.60 \%)$. The most frequent ages are between 0 to 10 years $(19.14 \%), 11$ to 20 years (12.88\%), and 41 to 50 years $(15.06 \%)$. The most common complaints are: Upper Respiratory tract infections (URTIs) had a total relative mean of $15.64 \%$; lumbago $10.35 \%$; asthmatic crisis 5.69\%. The most used drugs are: Dipyrone, total relative mean of 17.33\%; Dexamethasone 12.06\%; Diclofenac $8.74 \%$. The total relative average release rate is $95.80 \%$ while the total relative average hospitalizations are $4.20 \%$. It is concluded that, knowing the pharmacoepidemiological outline of a hospital environment is to be able to provide the entire staff of employees with the profile of patients admitted, as well as being the basis for the identification and
\end{abstract}


analysis of the real needs by the managers. However, it is necessary to emphasize that the study in question opens paths for the construction of future works, aimed at understanding the frequency of return of them to the emergency room referring to the same complaints, as well as the percentage of patients released, with drug therapy prescribed for later acquisition on an outpatient basis.

Keywords: Pharmacoepidemiology; Variables; Hospital; Managers.

\section{Resumen}

El objetivo de este estudio es cuantificar y presentar datos epidemiológicos que puedan dar un aporte a la gestión de un hospital del interior de Minas Gerais en el sentido de mejorar la calidad de los servicios que ofrecen. Este es un estudio observacional transversal, retrospectivo, con enfoque cualitativo y cuantitativo. El hospital es pequeño, tiene 46 camas hospitalarias. Por conveniencia, se utilizó la técnica de muestreo no probabilística. Para la recolección y análisis de los datos se utilizó como herramienta la versión Excel 2007. Las informaciones se obtuvieron utilizando las variables: edad, sexo, motivo de ingreso, medicamentos utilizados y resultados. La muestra analizada contiene 9.246 formularios de ingreso a urgencias. Entre estos, se incluyeron $1.328(14,36 \%)$ y se excluyeron $7.918(85,64 \%)$. El género femenino es mayoritario en el espacio muestral (61,39\%), a diferencia del masculino (38,60\%). La edad más frecuente es entre 0 a 10 años $(19,14 \%), 11$ a 20 años $(12,88 \%)$ y 41 a 50 años $(15,06 \%)$. Las quejas más comunes son: Infecciones de las vías respiratorias superiores (URTIs) con uno promedio relativo total de 15,64\%; Lumbalgia 10,35\%; Crisis asmática 5,69\%. Los fármacos más utilizados son: Dipirona, promedio relativo total de 17, $33 \%$; Dexametasona 12,06\%; Diclofenaco 8,74\%. El promedio total relativo a la tasa de liberación es $95,80 \%$; y el promedio total relativo a hospitalizaciones $4,20 \%$. Se concluye que, conocer el perfil farmacoepidemiológico de un entorno hospitalario es poder proporcionar a todos los empleados el perfil de los pacientes ingresados, además de ser la base para la identificación y análisis de las necesidades reales por parte de los responsables. Sin embargo, es necesario destacar que el estudio en cuestión abre caminos para la construcción de obras futuras, orientadas a comprender la frecuencia de retorno de los mismos a urgencias referidas a las mismas quejas, así como el porcentaje de pacientes dados de alta con tratamiento farmacológico prescrito para su posterior adquisición de forma ambulatoria.

Palabras clave: Farmacoepidemiología; Variables; Hospital; Gerentes.

\section{Introdução}

Os hospitais são instituições complexas, onde se fazem presentes equipes multiprofissionais que trabalham no sentido de tratar e prevenir danos à saúde. Nesse contexto, boas práticas de gestão é uma necessidade existente, na qual vários hospitais se mostram carentes. O gerenciamento de um ambiente hospitalar, seja ele de pequeno, médio e grande porte, constitui um grande desafio a ser superado na saúde, visto que para administrar um hospital exige certos conhecimentos acerca da regulação, dos financiamentos, bem como do manejo de recursos físicos, humanos e econômicos (Farias \& De Araujo, 2017).

No período entre 1964 a 1985 que compreendia a ditadura militar, a saúde por sua vez não era vista como direito social, e a mesma era promovida, mediante a três categorias; os que podiam pagar pelo serviço privado em saúde, os que tinham direito ao serviço público assegurados pela previdência social e, por fim os excluídos sem direito algum de usufruir dos serviços em saúde (Baldoni, 2011). Nessa perspectiva, a horizontalidade entre os pontos de atenção é um passo importante a ser dado para que as ações de promoção a saúde sejam trabalhadas de maneira fidedigna buscando a integralidade do cuidado (Da Silva, 2011).

A definição da premissa "acesso aos serviços de saúde" é complexa e está relacionada à percepção das carências existenciais de saúde, bem como, da conversão destas necessidades em demanda e, destas em uso (Ojanuga \& Gilbert, 1992). Elementos atrelados à oferta podem contribuir ou dificultar o acesso. Oferecer um serviço ao qual o indivíduo recorre constantemente quando necessita de cuidados em saúde, mostra-se associado ao uso, podendo ser considerado um indicador de acesso (Marcus \& Siegel, 1982; Puentes-Markides, 1992; Pinheiro et al., 2002). Assim sendo, o padrão de uso de serviços de saúde de um determinado perfil populacional é predominantemente explicado por seu perfil de necessidades em saúde (Hulka \& Wheat, 1985). 
Assim sendo, a atenção secundária ou especializada está inserida na rede de atenção saúde, oferecendo serviços a nível ambulatorial e hospitalar, mediante os procedimentos de média complexidade, os quais são; serviços médicos especializados, apoio diagnóstico e terapêutico e, atendimento de urgência e emergência (Brasil, 2010; Erdmann et al., 2013).

A farmacoepidemiologia é uma ciência que surgiu nos últimos 20 anos, a qual estuda as qualidades de consumo de medicamentos e seus efeitos na população em termos farmacológicos e epidemiológicos (Chauvin, 2002). Nesse sentido, além dessas citadas, existem mais três abordagens conceituadas como subáreas, sendo elas: a farmacovigilância, que expõem os eventos adversos a medicamentos, bem como as reações adversas a medicamento, a farmacoeconomia, expressa a relação entre o impacto econômico e os benefícios que o medicamento promove na saúde do paciente e, por fim os estudos de utilização de medicamentos, o qual lança mão de metodologias que possam analisar estudar os profissionais prescritores e a população que utilizam os medicamentos (Baldoni, 2011).

Defronte da necessidade de conhecer o perfil dos pacientes que usufrui dos serviços hospitalares, ficam as seguintes perguntas a serem respondidas. As perguntas a serem respondidas pela pesquisa são: Qual é a representatividade que um estudo farmacoepidemiológico pode fornecer para o ambiente hospitalar? Qual é a contribuição que a farmacoepidemiologia pode trazer para os gestores hospitalares?

Anteposto, este estudo apresenta como objetivo: Mensurar e apresentar dados epidemiológicos que possam contribuir na gestão de um hospital do interior de Minas Gerais, com intuito de aprimorar a qualidade dos serviços oferecidos.

\section{Metodologia}

Trata-se de um estudo com delineamento observacional do tipo transversal, retrospectivo com abordagem qualiquantitativa. Estudo observacional é um tipo de estudo que acompanha o objeto de estudo, sem intervir ou modificá-lo. A transversalidade é um segmento que o fator ou causa previamente exposto, se faz presente ao efeito no mesmo momento ou intervalo de tempo analisado. A análise de registros do passado até o presente é um tipo de direcionalidade temporal, definida como retrospecção (Li et al., 2017).

A amostragem não probabilística por conveniência se desenha a partir da escolha deliberada dos elementos que compõe a amostra, de acordo com critérios e julgamentos estabelecidos pelos pesquisadores, sendo estes os critérios para inclusão e exclusão (Vieira, 1997). E por fim, a pesquisa qualiquantitativa consiste na união de duas abordagens, a qualitativa

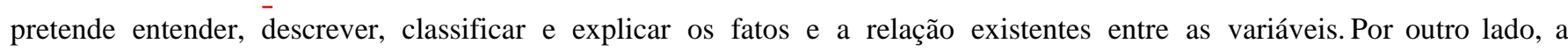
abordagem quantitativa tem como base a quantificação dos dados, para posterior análise e discussão (Santos, 1999).

O Local de pesquisa é um hospital público municipal, pertencente a uma cidade do interior de Minas Gerais, com população estimada de 13.600, segundo o ultimo senso do IBGE em 2020, a $763 \mathrm{Km}$ de Belo Horizonte MG. O hospital em questão é de pequeno porte, contendo 46 leitos, sendo três apartamentos cada qual contendo 2 leitos, o berçário neo natos com 2 leitos, a pediatria tendo 7 leitos, enfermaria masculina com 5 leitos, enfermaria feminina com 6 leitos, enfermaria cirúrgica com 6 leitos, maternidade com 6 leitos, pré-parto com 3 leitos e, o bloco de observação com 5 leitos. A população alvo do trabalho é constituída por fichas de pronto- atendimento de neonatos, recém nascidos, crianças, adolescentes e adultos, de ambos os sexos, os quais devidos algum motivo específico procuraram o pronto-socorro do referido local de pesquisa.

O instrumento para coleta de dados é composto pelas as fichas de admissão referente ao período entre 01/01/2019 a 31/11/2019, compreendendo o curso das estações que compõe o ano para o atendimento no pronto-socorro. De maneira a obter uma amostra que represente a população em estudo, utilizou-se a amostragem não probabilística por conveniência, identificando as fichas de admissão que atendem aos critérios de inclusão e exclusão para coletar os dados. O primeiro consiste em fichas que contenham apenas medicamentos disponíveis na unidade, fichas compostas por mais de um medicamento, bem 
como fichas ditas legíveis. O segundo é pautado em fichas de pacientes que não aguardaram o atendimento, fichas que há falta de informação, fichas que contenham apenas insumos hospitalares.

A ferramenta Excel versão 2007, foi utilizada para coleta e análisedos dados, compreendida a apreciação, através de tabelas e gráficos que representam dois meses de cada estação. As informações foram extraídas mediante as variáveis: Idade, sexo, motivo da admissão, medicamentos utilizados e desfechos. Esses compõem os indicadores, perfil populacional, perfil dos motivos de admissão, perfil medicamentoso e, respectivos desfechos clínicos. Contudo isso usou- se uma ferramenta que compõem o ramo da epidemiologia clínica, a frequência, sendo utilizada em todas as variáveis, bem como em todos os dados que fazem parte desta, tudo isso com a intenção de mensurar e avaliar com maior destreza as informações.

Todos os aspectos éticos dispostos na Resolução 466/2012 foram respeitados, sendo esta pesquisa aprovada pelo comitê de ética em pesquisa da Faculdade Independente do Nordeste, com parecer de aprovação № 4.482.996.

\section{Resultados e Discussão}

Após a etapa de coleta e análise dos dados como mostra a Tabela 1, a amostra contém 9.246 fichas de admissão para o pronto- atendimento, pertencente ao somatório de todas as estações. Dentre essas, foram incluídas $1.328(14,36 \%)$ e, excluídas 7.918 (85,64\%). Tendo como base o total de fichas incluídas, essas compõem o espaço amostral das quatro estações estudadas, se fragmentando em: Verão 515 (21,48\%); Outono 365 (14,95\%), Inverno 206 (9,36\%), Primavera 242 (10,97\%), bem como, as fichas excluídas, as quais se dissociam em: Verão 1.883 (78,52\%), Outono 2.441 (85,05\%), Inverno 2.201 (90,64\%), Primavera $2.206(89,03 \%)$.

Tabela 1. Frequência Absoluta e Relativa das Estações.

\begin{tabular}{lccccc}
\hline Estações & Incluídos & Excluídos & Amostra total & \multicolumn{2}{c}{ Incluídos (\%) Excluídos (\%) } \\
\hline Verão & 515 & 1883 & 2398 & $21,48 \%$ & $78,52 \%$ \\
Outono & 365 & 2076 & 2441 & $14,95 \%$ & $85,05 \%$ \\
Inverno & 206 & 1995 & 2201 & $9,36 \%$ & $90,64 \%$ \\
Primavera & 242 & 1964 & 2206 & $10,97 \%$ & $89,03 \%$ \\
Total & 1328 & 7918 & 9246 & $14,36 \%$ & $85,64 \%$ \\
\hline
\end{tabular}

Fonte: Dados da pesquisa.

Os resultados obtidos, descrevem as variáveis de acordo com cada estação, expressando também uma média relativa total dispostas nas Tabelas 2 a 5. Partindo do pressuposto, segundo a Tabela 1 é, possível evidenciar o decréscimo de dados incluídos, assim como a crescente taxa de informações excluídas por estações, ao decorrer do período analisado. Isso pode ser explicado, pelo enquadramento aos critérios de inclusão e exclusão, apoiado pela amostragem convencional. Através disso, a mesma foi aproveitada para filtrar dados, a fim de obter um espaço amostral que representa a população em estudo, retirando assim informações que poderiam contribuir para o enviesamento da pesquisa.

\subsection{Análise por idade e sexo}

A tabela abaixo divulga as frequências absolutas ( $\operatorname{Frq} A)$ e relativas ( $F r q R)$ de cada estação, alusivo a duas variáveis, sexo e idade. Além disso, a Tabela 2, também aponta o total amostral de fichas incluídas e analisadas das estações: Verão $\mathrm{n}=$ 515 , Outono $n=365$, Inverno $n=206$, Primavera $n=242$. Desse modo, é factível mencionar que a estação verão, possui a maior amostra do estudo, distintamente do inverno, que detém a menor amostra. 
Tabela 2. Análise das Variáveis Sexo e Idade.

\begin{tabular}{cccccc}
\hline Sexo & $\begin{array}{c}\text { Frq A/R- } \\
\text { Verão }\end{array}$ & $\begin{array}{c}\text { Frq A/R- } \\
\text { Outono }\end{array}$ & $\begin{array}{c}\text { Frq A/R- } \\
\text { Inverno }\end{array}$ & $\begin{array}{c}\text { Frq A/R- } \\
\text { Primavera }\end{array}$ & $\begin{array}{c}\text { Média } \\
\text { \% }\end{array}$ \\
\hline Feminino & $329(63,88 \%)$ & $226(61,92 \%)$ & $125(60,68 \%)$ & $143(59,09 \%)$ & $61,39 \%$ \\
Masculino & $186(36,12 \%)$ & $139(38,08 \%)$ & $81(39,32 \%)$ & $99(40,91 \%)$ & $38,60 \%$ \\
Total & $515(100 \%)$ & $365(100 \%)$ & $206(100 \%)$ & $242(100 \%)$ & \\
Idade & & & & & \\
0 a 10 & $86(16,70 \%)$ & $79(21,64 \%)$ & $43(20,87 \%)$ & $42(17,36 \%)$ & $19,14 \%$ \\
11 a 20 & $59(11,46 \%)$ & $41(11,23 \%)$ & $27(13,11 \%)$ & $38(15,70 \%)$ & $12,88 \%$ \\
21 a 30 & $70(13,59 \%)$ & $43(11,78 \%)$ & $25(12,14 \%)$ & $20(8,26 \%)$ & $11,44 \%$ \\
31 a 40 & $94(18,25 \%)$ & $56(15,34 \%)$ & $23(11,17 \%)$ & $30(12,40 \%)$ & $14,29 \%$ \\
41 a 50 & $72(13,98 \%)$ & $53(14,52 \%)$ & $33(16,02 \%)$ & $38(15,70 \%)$ & $15,06 \%$ \\
$51-60$ & $59(11,46 \%)$ & $39(10,68 \%)$ & $19(9,22 \%)$ & $30(12,40 \%)$ & $10,94 \%$ \\
61 a 70 & $37(7,18 \%)$ & $20(5,48 \%)$ & $18(8,74 \%)$ & $19(7,85 \%)$ & $7,31 \%$ \\
71 a 80 & $24(4,66 \%)$ & $27(7,40 \%)$ & $15(7,28 \%)$ & $16(6,61 \%)$ & $6,49 \%$ \\
81 a 90 & $14(2,72 \%)$ & $7(1,92 \%)$ & $3(1,46 \%)$ & $9(3,72 \%)$ & $2,45 \%$ \\
Total & $515(100 \%)$ & $365(100 \%)$ & $206(100 \%)$ & $242(100 \%)$ & \\
\hline
\end{tabular}

Fonte: Dados da pesquisa.

A Tabela 2, retrata certa linearidade associado a um decréscimo pouco significativo ao passo de uma estação a outra em relação a representatividade da presença feminina no serviço de pronto-atendimento, sendo possível observar que, o sexo feminino compõe a maior parte do espaço amostral, media relativa total 61,39\%, desigualmente o sexo masculino representa $38,60 \%$, visto que a estação verão teve maior frequência absoluta (Frq A) e relativa (Frq R) para o sexo feminino 329 (63,88\%) e, menor índice no inverno 125 (60,68\%). Diferente disso, o sexo masculino teve seu maior índice na estação primavera 99 $(40,91 \%)$ e, menor índice no verão 186 (36,12\%), quando comparado com as outras estações.

Continuamente, a Tabela 2 mostra que a média relativa de idade de maior frequência, está entre 0 a 10 anos de (19,14\%), 11 a $20 \operatorname{anos}(12,88 \%)$ e, 41 a 50 anos (15,06 \%). Diferente disso, as menores médias de faixas etárias, está entre 61 a $70 \operatorname{anos}(7,31 \%), 71$ a $80(6,49 \%)$ e, 80 a 90 anos (2,45\%). A tabela acima, também relata que, a faixa etária de 0 a 10 anos, teve seu maior índice, na estação outono 79 (21,64\%) e no inverno 43 (20,87\%) e, menor índice no verão 86 (16,70\%). Adicionalmente, o intervalo de idade entre 41- 50 anos de idade, teve sua maior expressividade no inverno 33 (16,22\%) e na primavera $38(15,70 \%)$, menor representatividade no verão 72 (13,98\%), isso tudo quando comparado com as outras estações.

Campos (2007) analisou 216 prontuários eletrônicos de pacientes acolhidos na unidade de pronto atendimento da emergência do Hospital de Base no intervalo de janeiro a dezembro de 2005, 61,5\% dos pacientes pertencia ao sexo feminino e, 38,5\% para o sexo masculino, estando assim de acordo com a tabela 2. Ademais, Olivati et al (2010) em seu estudo, mostra mediante a distribuição da amostra segundo o sexo, que a maior procura pelos atendimentos é exercida pelo sexo feminino $54,34 \%$ e de acordo com a faixa etária, o maior numero de pacientes assistidos tinha idade entre 18-29 anos, a medida que a faixa etária menos assistida era acima de sessenta anos, estando assim parcialmente de acordo com a tabela 2.

Nessa mesma perspectiva, Oliveira et al (2011), analisou 3.424 boletins de atendimento, dentre esses 53,7\% eram referentes à pacientes do sexo feminino e, 46,3\% do sexo masculino e, 75,5\% das fichas representavam pacientes de 14 a 54 anos, mostrando estar de acordo com a Tabela 2. Assim como, Dall'Agnol et al (2004), relataram que dos 48 pacientes que fizeram parte da pesquisa, $30(62,5 \%)$ eram mulheres, 18 (37.5\%) homens e, 25\% dos participantes apresentavam 65 anos ou mais, a primeira variável está em concordância com a Tabela 2, de modo que a segunda discorda 
Contudo, linhas de pesquisas que estudam as diferenças de gênero no âmbito da saúde em sociedades industrializadas, demonstram que embora as mulheres vivem mais que os homens, as mesmas relatam mais morbidade, bem como problemas psicológicos atrelado ao maior interesse das mesmas com a sua saúde e, assim utilizam mais serviços de saúde (Macintyre et al., 1996 \& 1999; Bird \& Rieker, 1999). Em contrapartida essa importante contradição decresce quando é analisado o padrão de doenças nas diferentes faixas etárias, sem pormenorizar. Os homens sofrem mais com doenças crônicas fatais (Isquemias do coração, aterosclerose, enfisema), oposto a isso as mulheres tendem a doenças agudas e transitórias (doenças das vias aéreas superiores, gastroenterites), bem como doenças crônicas não fatais (tireoidites, anemias, enxaqueca) (Verbrugge, 1989; Bird e Rieker, 1999).

\subsection{Análise por motivo de admissão}

Garantir o acesso aos serviços de saúde é promover a queda da morbidade e mortalidade. Haja vista que o indivíduo que acredita ter um problema de saúde será assistido pelo profissional, que irá ajudá-lo a compreender, se o problema é serio o bastante para que tenha atenção adicional, ou um problema autolimitado que não requer de atenção especial ( Starfield, 2002 ). Deste modo, as necessidades dos pacientes serão sanadas através de consultas, exames, terapia medicamentosa e, outros procedimentos, pois é assim que os estabelecimentos de saúde organizam seus serviços, com intuito de atender as queixas, elevando a qualidade de vida (Pinheiro, 2007). Consagradamente, no Brasil a atenção básica encontra dificuldades para a prestação de serviços de saúde, devido a isso a população acaba explorando os prontos- socorros, em busca de solução para os seus variados problemas, fazendo assim o mesmo de porta de entrada para o Sistema Único de Saúde (SUS) (Olivati et al., 2010).

A Tabela 3 mostra os principais motivos pelo qual o paciente procurou o serviço de atendimento no pronto socorro, de acordo com cada estação. Diante isso, é possível perceber que queixas como Infecções das Vias Aéreas Superiores (IVAS), tiveram uma média relativa total de $15,64 \%$, lombalgia $10,35 \%$, crise asmática $5,69 \%$, cefaléia 5,55\%, gastrite 4,50\%, crise hipertensiva 3,35\%, compondo a maior parte das ocorrências assistidas pelo local estudado.

Tabela 3. Análise da Variável Motivo de Admissão.

\begin{tabular}{|c|c|c|c|c|c|}
\hline Motivo de admissão & $\begin{array}{c}\text { Frq A/R- } \\
\text { Verão } \\
\end{array}$ & $\begin{array}{c}\text { Frq A/R- } \\
\text { Outono } \\
\end{array}$ & $\begin{array}{c}\text { Frq A/R- } \\
\text { Inverno } \\
\end{array}$ & $\begin{array}{c}\text { Frq A/R- } \\
\text { Primavera } \\
\end{array}$ & $\begin{array}{c}\text { Media } \\
\% \\
\end{array}$ \\
\hline Cefaléia & $35(6,8 \%)$ & $24(6,58 \%)$ & $8(3,88 \%)$ & $12(4,96 \%)$ & $5,55 \%$ \\
\hline Crise hipertensiva & $0(0,00 \%)$ & $24(6,58 \%)$ & $9(4,37 \%)$ & $6(2,48 \%)$ & $3,35 \%$ \\
\hline Crise asmática & $12(2,33 \%)$ & $14(3,83 \%)$ & $23(11,65 \%)$ & $12(4,95 \%)$ & $5.69 \%$ \\
\hline Dengue & $0(0,00 \%)$ & $21(5,75 \%)$ & $10(4,85 \%)$ & $0(0,00 \%)$ & $2,65 \%$ \\
\hline Enteroinfecção & $35(6,8 \%)$ & $13(3,56 \%)$ & $0(0,00 \%)$ & $0(0,00 \%)$ & $2,59 \%$ \\
\hline Gastrite & $27(5,24 \%)$ & $20(5,48 \%)$ & $15(7,28 \%)$ & $0(0,00 \%)$ & $4,50 \%$ \\
\hline IVAS & $59(11,45 \%)$ & $77(21,09 \%)$ & $27(13,10 \%)$ & $41(16,94 \%)$ & $15,64 \%$ \\
\hline Lombalgia & $39(7,57 \%)$ & $28(7,67 \%)$ & $25(12,14 \%)$ & $34(14,05 \%)$ & $10,35 \%$ \\
\hline
\end{tabular}

Fonte: Dados da pesquisa.

Ademais, os motivos de admissão descritos na Tabela 3 acima, tiveram comportamentos diferentes expressados pelas respectivas frequências absolutas (Frq A) e relativas (Frq R) nas quatro estações estudadas: IVAS teve sua maior participação no outono $77(21,09 \%), n=365$ e, menor participação no verão $59(11,45 \%), n=515$; cefaléia teve seu maior índice no verão 35 ( 6,18\%), $\mathrm{n}=515$ e, menor índice no inverno, $8(3,88 \%), \mathrm{n}=206$; lombalgia maior índice na primavera $34(14,05 \%), \mathrm{n}=242$ e, menor índice no verão 39 (7,57\%), n= 515; crise asmática maior índice no inverno $23(11,65 \%)$, n=206 e, menor índice no 
verão 12 (2,33\%), n= 515 ;gastrite maior índice no inverno 15 ( 7,28\%), n= 206, menor índice verão $27(2,24 \%), n=515$ e, não foi constatado índice na primavera e, por fim a crise hipertensiva teve sua maior expressividade no outono 24 (6,58\%), menor índice na primavera 6 ( $2,48 \%)$ e, não obteve índice no verão.

Em seu estudo, Olivati et al (2010), avaliou o perfil de demanda de um pronto-socorro no interior do estado de São Paulo, reportando os dados: doenças respiratórias $11,97 \%$, dor na coluna $8,89 \%$, dores gástricas $6,28 \%$ e, cefaléia $5,69 \%$, mostra estar de acordo com a Tabela 3, a qual mostra uma media relativa para Lombalgia de 10,35\%, gastrite 4,50\%, cefaléia $5,55 \%$ e, infecções das vias aéreas superiores (IVAS) de 15,64\%, aliado á eventos de crise asmática 5,69\%, compondo o grupo de doenças respiratórias. Todavia, dentre todas as ocorrências exibida por esse autor a, de maior índice é referente a acidentes gerais, $14,52 \%$, estando assim em desacordo com a (Tabela 3), que não menciona nenhum dado sobre tal eventualidade, bem como o trabalho não manifesta nenhuma informação sobre crises hipertensivas, enquanto a tabela 3 demonstra uma media relativa de 3,35\% para tal eventualidade associado ao seu maior índice no outono $24(6,58 \%)$.

Na sequência, Oliveira et al (2011), através do seu trabalho, descreve como queixas principais: cefaléia $316(9,22 \%)$, lombalgia $225(6,57 \%) \%)$, dor de garganta 109 (3,18\%), epigastralgia 103 (3,00\%), sendo essas, ocorrências condizentes com as informações que a Tabela 3 fornece. Analisando de acordo a sazonalidade, esse autor retrata que no verão as queixas de maior frequência foram lombalgia 78 (9,5\%), cefaléia $65(7,8 \%)$ e dor abdominal 43 (5,2\%); outono, cefaléia $102(12 \%)$, lombalgia 54 (6,6\%) e dor abdominal 39 (4,7\%); inverno, cefaléia 79 (9,1\%), tosse 58 (7\%) e, dor abdominal $50(6,1 \%)$, primavera, foram cefaléia 65 (7,9\%), dor torácica 49 (6\%) e lombalgia $48(6 \%)$. As queixas de cefaléia 65 (7,8\%) no verão e, lombalgia 54 (6,6\%) no outono, se assemelha aos dados da Tabela 3, que retrata cefaléia 35(6,8\%) no verão e, lombalgia 24 $(6,58 \%)$ no outono. É pertinente discutir também que, relatos de cefaléia 24 (6,58\%) no outono e, lombalgia 34 (14,05\%) na primavera, expressados pela Tabela 3, se diverge das informações do autor em questão.

Aliado a discussão anterior, os fatores sazonais como: precipitação, temperatura, umidade e velocidade do vento, têm grande ligação na incidência de infecções virais e, na prevalência de doenças do trato respiratório (Aykaç et al., 2018). As infecções do trato respiratório superior, estão presentes em sua maioria em países de zonas temperadas, sendo mais frequentes no outono e na primavera, tendo um aumento no inverno, isso devido a grande variação na mudança climática de cada estação ( Price \& Graham, 2019). Nesse caso, existem três teorias que explicam a sazonalidade viral. 1) Fator climático, o hospedeiro cria resistência pelo baixo nível de vitamina D, devido a falta de exposição ao sol, afetando assim diretamente na capacidade imunológica (Fisman, 2012; Martineau et al., 2017) . 2) Fatores meteorológicos, temperatura e umidade, tem efeito direto nas taxas de infecção (Fisman, 2012; Lofgren, 2007). 3) Fator comportamental, maior tempo em casa, agregação de pessoas, nos meses que a temperatura está baixa (Heikkinen \& Jârvinen, 2003; Fisman, 2012).

De 10 a $15 \%$ dos pacientes que procuram o pronto socorro, apresentam alguma condição neurológica, dentre esses 13 a 27,8\% queixam- se de cefaléia do tipo não traumática (Casado, 2011; Coban et al., 2016; Rizos et al., 2011). Esses dados representam 0,5 a 2,8\% dos atendimentos de emergência, embora aparentemente seja pouco frequente, essa eventualidade se torna um desafio para a consolidação do diagnóstico, devido à alta variabilidade de sinais e sintomas e, ao próprio ambiente de emergência, onde o atendimento é rápido e, ao uso dos recursos que auxiliam no diagnostico (Knox et al., 2012).

As doenças cardiovasculares refletem aproximadamente 17 milhões de mortes por ano, ao mesmo tempo que as complicações da Hipertensão Artéria Sistema (HAS), é responsável por 9,4 milhões destas mortes (OMS, 2013). A crise hipertensiva é uma importante complicação aguda da hipertensão, ocasionando assim admissão hospitalar de urgência (Johnson et al., 2012). A urgência hipertensiva está presente em 1 bilhão da população mundial, sendo responsável por 7,1 milhões de mortes no mundo por ano em média. Estima-se que quase 1\% dos pacientes com hipertensão evoluíram para uma crise hipertensiva futura (Varon, 2008 ). A rápida elevação da pressão arterial culminará em uma possível lesão vascular aguda dos órgãos vitais. Assim sendo, pode-se classificar como urgência hipertensiva quando não ocorre lesão de órgão alvo, 
diferentemente da emergência hipertensiva, que é marcada pela lesão de órgão alvo, evidenciando assim o risco de morte (Pierin et al., 2019).

\subsection{Análise por terapia medicamentosa}

O medicamento é considerado um bem essencial à saúde, uma importante ferramenta terapêutica, responsável por uma significativa melhora da qualidade e expectativa de vida da população. Mas, o seu uso irracional pode levar a diversas consequências em saúde, resultando em aumento dos gastos no âmbito da saúde (Arrais et al., 2005). Nos países desenvolvidos e subdesenvolvidos a utilização de medicamentos está em grande ascensão, isso pode ser explicado pelo aumento da expectativa de vida, tal como, o aumento dos gastos na área da saúde. As dificuldades orçamentárias do setor público têm levado os governantes a realizar reformas no setor. A consolidação do Sistema Único de Saúde (SUS) é, prejudicada por este impasse orçamentário (CONASS, 2003).

A Tabela 4, abaixo divulga os principais medicamentos utilizados para o manejo das ocorrências no serviço de urgência e emergência do local estudado. O espaço amostral de utilização de medicamento respectivo a cada estação é fragmentado em: Verão, $n=1380$; Outono, $n=1051$; Inverno, $n=475$; Primavera, $n=480$. Mas também, é possível constatar que os medicamentos como, dipirona, média relativa total de $17,33 \%$, dexametasona $12,06 \%$, diclofenaco $8,74 \%$; complexo B e vitamina C $5,74 \%$, atrovent e berotec $5 \%$, captopril $3,03 \%$, foram os que tiveram destaque no estudo.

Tabela 4. Análise da Variável Terapia Medicamentosa.

\begin{tabular}{cccccc}
\hline Medicamentos & $\begin{array}{c}\text { Frq A/R- } \\
\text { Verão }\end{array}$ & $\begin{array}{c}\text { Frq A/R- } \\
\text { Outono }\end{array}$ & $\begin{array}{c}\text { Frq A/R- } \\
\text { Inverno }\end{array}$ & $\begin{array}{c}\text { Frq A/R- } \\
\text { Primavera }\end{array}$ & $\begin{array}{c}\text { Media } \\
\text { \% }\end{array}$ \\
\hline Atrovent+ berotec & $30(2,17 \%)$ & $37(3,52 \%)$ & $47(9,89 \%)$ & $21(4,38 \%)$ & $5,00 \%$ \\
Bromoprida & $46(3,33 \%)$ & $22(2,09 \%)$ & $0,00 \%$ & $11(2,29 \%)$ & $1,92 \%$ \\
Buscopam Comp & $69(5 \%)$ & $35(3,33 \%)$ & $29(6,11 \%)$ & $20(4,17 \%)$ & $4,65 \%$ \\
Captopril & $28(2,03 \%)$ & $25(2,38 \%)$ & $15(3,16 \%)$ & $22(4,58 \%)$ & $3,03 \%$ \\
Comp B + Vit C & $128(9,28 \%)$ & $69(6,57 \%)$ & $16(3,37 \%)$ & $18(3,75 \%)$ & $5,74 \%$ \\
Dexametasona & $196(14,2 \%)$ & $111(10,57 \%)$ & $63(13,26 \%)$ & $49(10,21 \%)$ & $12,06 \%$ \\
Diclofenaco & $98(7,1 \%)$ & $62(5,9 \%)$ & $45(9,47 \%)$ & $60(12,5 \%)$ & $8,74 \%$ \\
Dipirona & $207(15 \%)$ & $184(17,51 \%)$ & $78(16,42 \%)$ & $98(20,42 \%)$ & $17,33 \%$ \\
Dexcloferinamina & $31(2,25 \%)$ & $38(3,62 \%)$ & $10(2,11 \%)$ & $0,00 \%$ & $1,99 \%$ \\
Metoclopramida & $32(2,32 \%)$ & $34(3,14 \%)$ & $11(5,89 \%)$ & $16(3,33 \%)$ & $3,67 \%$ \\
Ranitidina & $35(2,54 \%)$ & $0,00 \%$ & $14(2,95 \%)$ & $0,00 \%$ & $5,49 \%$ \\
SF 0,9\% & $76(5,51 \%)$ & $79(7,52 \%)$ & $30(6,32 \%)$ & $47(9,79 \%)$ & $7,28 \%$ \\
Simeticona & $39(2,83 \%)$ & $0,00 \%$ & $0,00 \%$ & $0,00 \%$ & $0,70 \%$ \\
\hline
\end{tabular}

Fonte: Dados da pesquisa.

Realizando uma análise por estações, a Tabela 4 acima, fornece valores absolutos e relativos de cada medicamento nas quatro estações que compõe o campo amostral do estudo. Assim sendo, é possível perceber que cada medicamento teve um comportamento diferente nas estações, mediante as respectivas frequências absoluta (Frq A) e relativa (Frq R). Podemos destacar que a dipirona teve seu maior destaque na primavera $98(20,42 \%), n=480$ e, menor índice no verão $207(15 \%), n=$ 1380, dexametasona, maior índice no verão $196(14,2 \%), \mathrm{n}=1380 \mathrm{e}$, menor índice na primavera $49(10,21 \%), \mathrm{n}=480$, diclofenaco, maior índice na primavera $60(12,5 \%), \mathrm{n}=480 \mathrm{e}$, menor índice no outono $62(5,9 \%), \mathrm{n}=1051$, complexo B e vitamina C, maior índice no verão 128 (9,28\%), n= 1380 e, menor índice no inverno 16 ( 3,37\%), n= 475 , atrovent e berotec, 
maior índice no inverno $47(9,89 \%), \mathrm{n}=475 \mathrm{e}$, menor índice no verão $30(2,17 \%), \mathrm{n}=1380$, captopril, maior índice na primavera $22(4,58 \%), n=480 \mathrm{e}$, menor no verão índice $28(2,03 \%), n=1380$.

As unidades públicas contam com uma gama de medicamentos ditos analgésicos, pertencente a família dos não esteroidais, corticosteróides, neurolépticos e opioides, sendo o mais comum deles a dipirona e, não menos importante em segundo lugar o diclofenaco, o qual é pertencente a classe dos antiinflamatórios não esteróides (AINES). Esses antiinflamatórios são as drogas mais utilizadas nesses ambientes (Rabello, 1997; Bigal et al., 1999). Segundo Bigal et al (2002), dos 1.254 pacientes que foram tratados para cefaléia, 64 (29,7\%) deles necessitaram de hospitalização. Desses, 83,6 \% melhoraram com dipirona intravenosa e, 66,7\% com diclofenaco intramuscular. Portanto esses dados se assemelham com as infomações da Tabela 4, onde a mesma anuncia a soberania da dipirona, oposto disso o diclofenaco que não excerce liderança, mas está no top três de usos perdendo apenas para a dexametasona, um corticoide esteroidal. Essa, também, se mostrou amplamente utilizada, visto que teve bons desfechos no manejo da enxaqueca, cefaleia e, crises asmáticas quando associado a outro medicamento, explicado pela a sua dupla ação imunomoduladora e imunosupressora (Orr et al., 2016; Kirkland, 2018).

Micronutrientes como, por exemplo, vitaminas do complexo B e a vitamina C, desempenham no organismo papel importante fisiologicamente e bioquimicamente, podendo exercer tanto ação oxidante como a anti-oxidante, contribuindo para o anabolismo e catabolismo protéico, contribuindo no fortalecimento do sistema imunológico (Tardy et al., 2020). De acordo com Gaby (2002), vitaminas do complexo B e vitamina C, demonstram ser efetivos como adjuvante contra crises asmáticas agudas, enxaquecas, mialgias, Infecções do trato respiratório superior e inferior. Nessa situação, é cabível mencionar que o autor em questão concorda com a Tabela 4, uma vez que o polivitaminico possui uma media relativa significativamente relevante.

As afecções do trato respiratório inferior estão cada vez mais presentes nas ocorrências dos pronto-socorros, e sendo grande proporcionadores de internações hospitalares, principalmente nos meses em que o inverno é presente. A asma aguda é personagem de destaque nesse importante grupo de enfermidades, e 22\% dos casos estão presentes na America latina, dos quais acabam em hospitalização (Kirklan et al., 2017). Em sua pesquisa, Rebuck et al (1987), avaliava a eficácia dos anticolinérgicos e simpaticomiméticos, isoladamente ou em associação, numa amostra de 148 pacientes com crise asmática aguda, tanto o bromidrato de fenoterol, quanto o brometo de ipatrópio, contribuíram para uma melhora significativa, mas quando os mesmos foram utilizados de maneira associada, resultaram em desfechos significativamente maiores. A partir da luz das informações é visível certa sincronia com a Tabela 4, uma vez que as afecções respiratórias são geralmente sazonais.

Os inibidores da enzima conversora de angiotensina 2, são medicamentos amplamente utilizados para o tratamento da hipertensão Arterial Sistêmica (HAS) a nível ambulatorial. Nos atendimentos de urgência e emergência dos hospitais afins, é cada vez mais comum a entrada de pacientes com crise hipertensiva, diagnosticados ou não com HAS. Os médicos plantonistas rotineiramente lançam mão do uso off-label do captopril, via sub-lingual, para sanar o pico hipertensivo (Salkic, 2015). Contudo isso, Biollaz (1983), avaliou o valor hipotensor do captopril, frente a uma crise hipertensiva em 9 pacientes, não diagnoticados com HAS. Houve uma redução de 12/4 mmHg após $30 \mathrm{~min}$ da adiministração de $25 \mathrm{mg}$ de captopril via sublingual. Dessa forma, os autores comprovaram o valor de indicação, efetividade e, segurança do captopril, justificando o seu uso, estando assim em conformidade com a Tabela 4.

\subsection{Análise por desfecho}

Os desfechos em saúde de um hospital irão se basear nos tipos de serviços existentes a serem oferecidos ao paciente, bem como dependerá do perfil de pacientes assistidos pela unidade em questão. Essas duas variáveis são responsáveis pelos cincos, desfechos possíveis que acontecem na rotina de um hospital, sendo eles liberação, observação, internação, transferências e os óbitos (Ramos et al., 2015). 
Neste caso, a Tabela 5 aponta duas variáveis, à liberação e internação, dentro das cinco mencionadas anteriormente. A média relativa total da taxa de liberação é de $95,80 \%$, diferentemente da média relativa total de internações, sendo de $4,20 \%$. e acordo com Oliveira et al., (2011), a maioria dos pacientes receberam alta hospitalar 74,1\%, seguidos de encaminhamentos 24,4\% para outras unidades e, internações representada por $0,2 \%$ dos pacientes atendidos. Com base nisso, percebe-se maior presença do índice de liberação, aliado ao baixo índice de internação, marcando concordância entre o autor e a, tabela abaixo.

Tabela 5. Análise da Variável Desfecho.

\begin{tabular}{cccccc}
\hline Desfecho & $\begin{array}{c}\text { Frq A/R } \\
\text { Verão }\end{array}$ & $\begin{array}{c}\text { Frq A/R } \\
\text { Outono }\end{array}$ & $\begin{array}{c}\text { Frq A/R } \\
\text { Inverno }\end{array}$ & $\begin{array}{c}\text { Frq A/R } \\
\text { Primavera }\end{array}$ & $\begin{array}{c}\text { Média } \\
\%\end{array}$ \\
\hline Liberação & $494(95,92 \%)$ & $352(96,44 \%)$ & $199(96,6 \%)$ & $228(94,21 \%)$ & $95,80 \%$ \\
\hline Internação & $21(4,08 \%)$ & $13(3,56 \%)$ & $7(3,4 \%)$ & $14(5,79 \%)$ & $4,20 \%$ \\
\hline Total & $515(100 \%)$ & $365(100 \%)$ & $206(100 \%)$ & $242(100 \%)$ \\
\hline
\end{tabular}

Fonte: Dados da pesquisa.

Diante dos dados da Tabela 5 acima, tanto a variável liberação, quanto á internação se comportaram de maneira distintas em cada estação, apontados pelas respectivas frequências absoluta (Frq A) e relativa (Frq R). É possível salientar que a variável liberação teve seu maior índice no inverno $199(96,6 \%)$ e, n=206 e, menor índice na primavera 228 $(94,21 \%)$ e, n= 242. Distintamente a isso, a variável internação teve seu maior índice na primavera $14(5,79 \%)$ e, n= 242 e, menor índice no inverno $7(3,4 \%), n=206$.

Os altos níveis de liberação e baixos níveis de internação são explicados pela, 1) alta frequência de ocorrências de baixa complexidade, devido a carência de uma Unidade de Saúde da Família (USF), em um bairro que fica nas proximidades do hospital, 2) desprovimento de estrutura física ideal para atender demandas de media e alta complexidade, 3) carência de recursos econômicos, visto que o local de pesquisa carece de profissionais médicos especialistas disponíveis em tempo integral, como também necessita de medicamentos, insumos hospitalares, aparelhagem especificas para realização de procedimentos de media e alta complexidade. Essa situação carencial irá resultar em regulação dos pacientes para unidades mais próximas, localizadas nas cidades circunvizinhas.

\section{Conclusão}

Conclui-se que, conhecer o traçado farmacoepidemiológico de um ambiente hospitalar, é poder fornecer a todo corpo de funcionários o perfil de paciente que os mesmos estão lidando diariamente ao longo da rotina de trabalho. Através do conhecimento quanto à faixa etária, sexo, motivo de admissão, terapia medicamentosa e, desfechos de maior frequência. Essas variáveis exercer papel importante na elaboração e, aplicação de protocolos, procedimentos operacionais padrão (POPs), resoluções diretoria colegiada (RDCs) e, portarias, a fim de aumentar a qualidade dos serviços prestados, tal qual também, serve de base para identificação e análise das reais necessidades, resolução de problemas, manejo racional dos recursos econômicos, tecnológicos e humanos por parte dos gestores. Isso tudo com o objetivo de fazer com que o estabelecimento de saúde trabalhe em função do paciente, promovendo melhoria na qualidade de vida e, contribui na redução do sucateamento do Sistema Único de Saúde (SUS).

Entretanto é necessário salientar que o estudo em questão abre caminhos para a construção de futuros trabalhos, direcionado ao entendimento, quanto à frequência de retorno dos mesmos ao pronto-socorro, referindo as mesmas queixas, bem como o percentual de pacientes liberados, com terapia medicamentosa prescrita para posterior aquisição a nível ambulatorial. Esses irão somar positivamente para a melhoria ou adesão de condutas médico hospitalares, introdução de 
métodos gerenciais de cunho racional, contratação do responsável técnico farmacêutico, criação da comissão de farmácia terapêutica (CFT), padronização farnacoterapêutica, tudo isso com objetivo de sanar as queixas dos pacientes.

\section{Referências}

Arrais, P. S. D., Brito, L. L., Barreto, M. L., \& Coelho, H. L. L. (2005). Prevalência e fatores determinantes do consumo de medicamentos no Município de Fortaleza, Ceará, Brasil. Cadernos de Saúde Pública, 21(6), 1737-1746. https://doi.org/10.1590/s0102-311x2005000600021

Aykaç, K., Karadağ-öncel, E., Bayhan, C., Tanır-Başaranoğlu, S., Akın, M. Ş., Özsürekci, Y., Alp, A., Cengiz, A. B., Kara, A., \& Ceyhan, M. (2018). Prevalence and seasonal distribution of viral etiology of respiratory tract infections in inpatients and outpatients of the pediatric population: 10 year follow-up. Turkish Journal of Pediatrics, 60(6), 642-652. 10.24953/TURKJPED.2018.06.004

Baldoni, A. (2011). A Farmacoepidemiologia No Brasil: Estado Da Arte Da Produção Científica. Revista Da Universidade Vale Do Rio Verde, 9(1), 7888. http://dx.doi.org/10.5892/ruvrd.v9i1.79

Bird, C. E., \& Rieker, P. P. (1999). Gender matters: an integrated model for understanding men's and women's health. Social science \& medicine, 48(6), 745755. https://doi.org/10.1016/S0277-9536(98)00402-X

Bigal, M. E., Bordini, C. A., \& Speciali, J. G. (1999). Tratamento da cefaléia em uma unidade de emergência da cidade de Ribeirão Preto. Arquivos de Neuropsiquiatria, 57(3B), 813-819. https://doi.org/10.1590/S0004-282X1999000500013

Bigal, M. E., Bordini, C. A., \& Speciali, J. G. (2002). Dipirona intravenosa para o tratamento agudo da cefaléia do tipo tensional episódica: um estudo randomizado, controlado por placebo, duplo-cego. Revista Brasileira de Pesquisas Médicas e Biológicas, 35 (10), 11391145. HTTPS://DX.DOI.ORG/10.1590/S0100-879X2002001000005

Biollaz, J, Waeber, B, Brunner, H. R. (1983) .Hypertensive crisis treated with orally administered captopril. Eur J Clin Pharmacol. (2):145-9. https://doi.org/10.1007/BF00543783

Brasil (2010). Portarian 4.479? https://conselho.saude.gov.br/ultimas_noticias/2011/img/07_jan_portaria4279_301210.pdf

Casado, V. (2011). Atención al paciente neurológico en los Servicios de Urgencias. Revisión de la situación actual en España. Neurología, 26 (4 ), $193-256$. https://doi.org/10.1016/j.nrl.2010.07.033

Chauvin, C., Madec, F., Guittet, M., \& Sanders, P. (2002). Pharmaco-epidemiology and-economics should be developed more extensively in veterinary medicine. Journal of veterinary pharmacology and therapeutics, 25(6), 455-459. https://doi.org/10.1046/j.1365-2885.2002.00443.x

Campos, J. F., Poletti, N. A., Rodrigues, C. D., Garcia, T. P., Angelini, J. F., Von Dollinger, A. P. A., \& Ribeiro, R. C. H. M. (2007). Trauma em idosos atendidos no pronto atendimento da emergência do Hospital de Base. Arq Ciênc Saúde, 14(4), 193-7. http://repositorio-racs.famerp.br/racs_ol/vol-144/ID214.pdf

Dall'Agnol, R. S. A., Albring, D. V., De Castro, M. S., \& Heineck, I. (2004). Problemas relacionados com medicamentos em serviço de Emergência de Hospital Universitário do Sul do Brasil. Estudo piloto. Acta Farmaceutica Bonaerense, 23(4), 540-545. http://www.latamjpharm.org/trabajos/23/4/LAJOP_23_4_5_1_F015YP8C7F.pdf

Coban, E., Mutluay, B., Sen, A., Keskek, A., Atakl, D., \& Soysal, A. (2016). Características, diagnóstico e evolução dos pacientes encaminhados a um prontosocorro neurológico especializado: estudo prospectivo observacional. Annals of Saudi medicine, 36 (1), 51-56. https://doi.org/10.5144/0256-4947.2016.51

Conselho Nacional de Secretários de Saúde (CONASS). (2003). A saúde na opinião dos brasileiros: um estudo prospectivo. Conselho Nacional de Secretários de Saúde. http://bvsms.saude.gov.br/bvs/publicacoes/opiniao_brasileiros1.pdf

Da Silva, S. F. (2011). Organização de redes regionalizadas e integradas de atenção à saúde: Desafios do Sistema Único de Saúde (Brasil). Ciencia e Saude Coletiva, 16(6), 2753-2762. https://www.scielosp.org/pdf/csc/2011.v16n6/2753-2762/pt

Erdmann, A. L., Andrade, S. R. de, Mello, A. L. S. F. de, \& Drago, L. C. (2013). A atenção secundária em saúde: melhores práticas na rede de serviços. Revista Latino-Americana De Enfermagem, 21(spe), 131-139. https://doi.org/10.1590/S0104-11692013000700017

Farias, D. C., \& De Araujo, F. O. (2017). Gestão hospitalar no brasil: Revisão da literatura visando ao aprimoramento das práticas administrativas em hospitais. Ciencia e Saude Coletiva, 22(6), 1895-1904. https://doi.org/10.1590/1413-81232017226.26432016

Fisman, D. (2012). Sazonalidade das infecções virais: mecanismos e incógnitas. Clinical Microbiology and Infection, 18 (10), 946-954. 10.1111 / J.14690691.2012 .03968

Fenves, A. Z., \& Ram, C. V. (2005). Drug treatment of hypertensive urgencies and emergencies. Semin Nephrol. (4), 272-80. https://doi.org/10.1016/j.semnephrol.2005.02.011

Gaby AR. (2002). Intravenous nutrient therapy: the "Myers' cocktail". Altern Med Rev. (5), 389-403. https://loudounholistichealthpartners.com/wpcontent/uploads/2017/02/Myers-Cocktail.pdf

Heikkinen, T., \& Järvinen, A. (2003). O frio comum. Lancet (Londres, Inglaterra) , 361 (9351), 51-59. https://doi.org/10.1016/S0140-6736(03)12162-9

Hulka, B., \& Wheat, J. (1985). Patterns of Utilization: The Patient Perspective. Medical Care, 23(5), 438-460. http://www.jstor.org/stable/3764983

Johnson W., Nguyen M. L, \& Patel R. (2012) .Hypertension crisis in the emergency department. Cardiol Clin. (4), 533-43. http://dx.doi.org/10.1016/j.ccl.2012.07.011 
Li, T., Xie, C., \& Jiao, H. (2017). Assessing fit of alternative unidimensional polytomous IRT models using posterior predictive model checking. Psychological Methods, 22(2), 397-408. https://doi.org/10.1037/met0000082

Lofgren, E., Fefferman, N. H., Naumov, Y. N., Gorski, J., \& Naumova, E. N. (2007). Sazonalidade da influenza: causas subjacentes e teorias de modelagem. Journal of virology, 81 (11), 5429-5436. https://doi.org/10.1128/JVI.01680-06

Macintyre S, Hunt K, Sweeting H. (1996). Gender differences in health: are things really as simple as they seem? Soc Sci Med 42(4):617-24. 10.1016/02779536(95)00335-5.

Macintyre S, Ford G, Hunt K. (1999). Do women 'over-report' morbidity? Men's and women's responses to structured prompting on a standard question on long standing illness. Soc Sci Med 48(1):89-98. 10.1016/s0277-9536(98)00292-5.

Marcus, A., \& Siegel, J. (1982). Sex Differences in the Use of Physician Services: A Preliminary Test of the Fixed Role Hypothesis. Journal of Health and Social Behavior, 23(3), 186-197. https://doi.org/10.2307/2136628

Martineau, A. R., Jolliffe, D. A., Hooper, R. L., Greenberg, L., Aloia, J. F., Bergman, P., Dubnov-Raz, G., Esposito, S., Ganmaa, D., Ginde, A. A., Goodall, E. C., Grant, C. C., Griffiths, C. J., Janssens, W., Laaksi, I., Manaseki-Holland, S., Mauger, D., Murdoch, D. R., Neale, R., Rees, J. R., \&Camargo, C. A., Jr (2017). Vitamin D supplementation to prevent acute respiratory tract infections: systematic review and meta-analysis of individual participant data. BMJ (Clinical research ed.), 356, i6583. https://doi.org/10.1136/bmj.i6583

Olivati, F. N., Brandão, G. A. M., Vazquez, F. L., Paranhos, L. R., \& Pereira, A. C. (2010). Perfil da demanda de um pronto-socorro em um município do interior do estado de São Paulo. Perfil Da Demanda de Um Pronto-Socorro Em Um Município Do Interior Do Estado de São Paulo, 15(3), 245-250. https://doi.org/10.5335/rfo.v15i3.1669

Oliveira, G. N., Silva, M. de F. N., Araujo, I. E. M., \& Filho, M. A. C. (2011). Perfil da população atendida em uma unidade de emergência referenciada. Revista Latino-Americana de Enfermagem, 19(3), 548-556. https://doi.org/10.1590/S0104-11692011000300014

Ojanuga, D. N., \& Gilbert, C. (1992). Women's access to health care in developing countries. Social science \& medicine, 35(4), 613-617. https://doi.org/10.1016/0277-9536(92)90355-T

Organização Mundial da Saúde (OMS). (2013). Um Resumo Global sobre Hipertensão: Silent Killer, Global Public Health Crisis? www.who.int/cardiovascular_diseases/publications/global_brief_hypertension.pdf .

Orr, S. L., Friedman, B. W., Christie, S., Minen, M. T., Bamford, C., Kelley, N. E., \& Tepper, D. (2016). Management of Adults With Acute Migraine in the Emergency Department: The American Headache Society Evidence Assessment of Parenteral Pharmacotherapies. Headache. (6):911-40. 10.1111/HEAD.12835.

Price, R., Graham, C., \& Ramalingam, S. (2019). Associação entre sazonalidade viral e fatores meteorológicos. Relatórios científicos, 9 (1), 929.

Pinheiro, R. (2007). Integralidade em saúde. Ciência \& Saúde Coletiva, 12(3), 553-565. https://www.epsjv.fiocruz.br/upload/d/integralidade.pdf

Pierin, A., Flórido, C. F, \& Santos, J. D. (2019). Crise hipertensiva: características clínicas de pacientes com urgência, emergência e pseudocrise hipertensiva em um pronto-socorro público. Einstein (São Paulo, Brasil), 17 (4), eAO4685.

Price, R., Graham, C., \& Ramalingam, S. (2019). Associação entre sazonalidade viral e fatores meteorológicos. Relatórios científicos, 9 (1), 929. https://doi.org/10.1038/s41598-018-37481-y

Rabello, G. D. (1997). Aspectos clínicos e terapêuticos das cefaléias agudas. Medicina (Ribeirão Preto), 30(4), 458-471. https://doi.org/10.11606/issn.21767262.v30i4p458-471

Rizos T., Jüttler E., Sykora M., Poli S., \& Ringleb P. A. (2011). Common disorders in the neurological emergency room--experience at a tertiary care hospital. Eur J Neurol. 18(3):430-5. HTTPS://DOI: 10.1111/J.1468-1331.2010.03170.X.

Rebuck, A. S., Chapman, K. R., Abboud, R., Pare, P. D., Kreisman, H., Wolkove, N., \& Vickerson, F. (1987). Nebulized anticholinergic and sympathomimetic treatment of asthma and chronic obstructive airways disease in the emergency room. Am J Med. (1):59-64. 10.1016/0002-9343(87)90378-0.

Ramos, M. C., da Cruz, L. P., Kishima, V. C., Pollara, W. M., de Lira, A. C., \& Couttolenc, B. F. (2015).Performance evaluation of hospitals that provide care in the public health system, Brazil. Rev Saude Publica; 49:43. 10.1590/S0034-8910.2015049005748.

Santos, S. (1999). Métodos qualitativos e quantitativos na pesquisa biomédica. J. Pediatr. (Rio J.),75(6), 401-406. http://www.jped.com.br/conteudo/99-7506-401/port.pdf

Salkic, S., Brkic, S., Batic-Mujanovic, O., Ljuca, F., Karabasic, A., \& Mustafic, S. (2015). Tratamento em pronto-socorro de crises hipertensivas. Arquivos médicos (Sarajevo, Bósnia e Herzegovina) , 69 (5), 302-306. https://doi.org/10.5455/medarh.2015.69.302-306

Starfield, B. (2006). Atenção primária: equilíbrio entre necessidades de saúde, serviços e tecnologia. https://unesdoc.unesco.org/ark:/48223/pf0000130805

Tardy, A. L., Pouteau, E., Marquez, D., Yilmaz, C., \& Scholey, A. (2020). Vitamins and Minerals for Energy, Fatigue and Cognition: A Narrative Review of the Biochemical and Clinical Evidence. Nutrients, 12(1), 228. https://doi.org/10.3390/nu12010228

Verbrugge, L. M. (1989). The twain meet: empirical explanations of sex differences in health and mortality. J Health Soc Behav (3):282-304. https://doi.org/10.2307/2136961

Vieira, S. (1997). Introdução à bioestatística. Elsevier Brasil, 2011. 
Research, Society and Development, v. 10, n. 7, e21910716519, 2021

(CC BY 4.0) | ISSN 2525-3409 | DOI: http://dx.doi.org/10.33448/rsd-v10i7.16519

Kirkland, SW, Cross, E., Campbell, S., Villa-Roel, C., \& Rowe, BH (2018). Corticosteroides intramusculares versus orais para reduzir as recidivas após a alta do departamento de emergência por asma aguda. $O$ banco de dados Cochrane de revisões sistemáticas, 6(6), CD012629. https://doi.org/10.1002/14651858.CD012629.pub2

Kirkland, S. W., Vandenberghe, C., Voaklander, B., Nikel, T., Campbell, S., \& Rowe, B. H. (2017). Agonistas beta-agonistas e anticolinérgicos inalatórios combinados para tratamento de emergência em adultos com asma. $O$ banco de dados Cochrane de revisões sistemáticas, 1 (1), CD001284. https://doi.org/10.1002/14651858.CD001284.pub2

Knox, J., Chuni, C., Naqvi, Z., Crawford, P., \& Waring, W. (2012). Presentations to an acute medical unit due to headache: a review of 306 consecutive cases. Acute Med. 11(3), 144-9. https://europepmc.org/article/med/22993744?utm_medium=email\&utm_source=transaction 